\title{
Neutrino opacities in a relativistic non interacting neutron gas
}

\section{K. Vantournhout*}

Ghent University, Department of Subatomic and Radiation Physics

E-mail: Klaas.Vantournhout@ugent.be

\section{N. Jachowicz}

Ghent University, Department of Subatomic and Radiation Physics

\section{J. Ryckebusch}

Ghent University, Department of Subatomic and Radiation Physics

We investigate the influence of relativity for the computation of neutrino mean-free paths in nuclear matter. This is done under the assumption that the nuclear matter is a non-interacting Fermi gas in beta-equilibrium. We show that relativity causes a considerable enhancement of neutrinoscattering cross-sections in neutron matter.

International Symposium on Nuclear Astrophysics - Nuclei in the Cosmos - IX

June 25-30 2006

CERN, Geneva, Switzerland

\footnotetext{
* Speaker.
} 


\section{Introduction}

Neutrino processes play a leading role in a wide variety of astrophysical phenomena. They witnessed nuclear processes during the Big Bang and became the famous messengers from the sun. These ghostly particles are also recognised as the representatives from distant stars. Reaching us from the centre of an exploding star, neutrinos are the first heralds announcing the end of the life of a massive star [1]. Infact, their impact on the dynamics of a type-II supernova is crucial for the fate of the event. In the same course of action, they play also a pivotal role in the first cooling stages of a neutron star during the first hundreds of years after its birth.

For a better understanding of the evolutionary stages of these events, it is necessary to have a detailed knowledge of neutrino transport properties in high density matter. Neutrino transport in supra-nuclear matter has been studied in a variety of models which take the correlations into account. RPA, molecular dynamics and Hartree analysis are some of the used techniques [2, 3, 4]. In our work we focus on the impact of the implementation of relativistic effects on the description of the process. A fully relativistic description of neutrino opacity's in neutron matter will be compared with its non-relativistic counterpart. To this purpose, neutron matter will be described as a noninteracting Fermi gas in $\beta$-equilibrium.

\section{Three quantities to look at}

When studying the influence of relativity in neutrino opacities in neutron matter, it is important to introduce three important quantities, namely the differential cross section, the phase space factor and the dynamic form factor.

The differential cross section for the interaction of a neutrino $v\left(p_{v}\right)$ with a hadron $h_{1}\left(p_{h_{1}}\right)$ in the relativistic gas can be written as

$$
\begin{aligned}
\mathscr{N} d^{6} \sigma & =\sum_{\substack{\alpha_{\nu}, \alpha_{h_{1}}, \alpha_{\ell}, \alpha_{h_{2}}}} \int F_{\alpha_{h_{1}}}^{h_{1}}\left(\vec{p}_{h_{1}}\right) d^{3} \vec{p}_{h_{1}} \delta^{4}\left(p_{v}+p_{h_{1}}-p_{\ell}-p_{h_{2}}\right) \frac{(2 \pi)^{10}}{v_{r e l}}\left|M\left(v, h_{1} \rightarrow \ell, h_{2}\right)\right|^{2} \\
& \times\left(1-F_{\alpha_{h_{2}}}^{h_{2}}\left(\vec{p}_{h_{2}}\right)\right) d^{3} \vec{p}_{h_{2}}\left(1-F_{\alpha_{\ell}}^{\ell}\left(\vec{p}_{\ell}\right)\right) d^{3} \vec{p}_{\ell},
\end{aligned}
$$

where the reaction products are denoted by $\ell\left(p_{\ell}\right)$ for the outgoing lepton, and $h_{2}\left(p_{2}\right)$ for the final nucleon, $v_{r e l}$ is the relative velocity of the incident particles. The probability distributions are represented by the Fermi distributions $F_{\alpha_{a}}^{a}\left(\vec{p}_{a}\right)$, the quantum numbers $\alpha_{a}$ identify the state particle $a$ is occupying. The Fermi distributions are related through the restrictions imposed by the betaequilibrium conditions for the $n, p$ and $e^{-}$in the gas. The dynamics of the interaction is contained in the matrix element $M\left(v, h_{1} \rightarrow \ell, h_{2}\right)$ that is calculated in first-order perturbation theory, using the full expression for the hadron vertex-function as given by [5]. The normalisation factor $\mathscr{N}$ is determined such that Eq. (2.1) represents the scattering cross-section per nucleon.

The differential phase space factor is then obtained when removing the dynamics of the interaction out of the differential cross-section.

$$
\begin{aligned}
\mathscr{N} d^{6} \Phi & =\sum_{\substack{\alpha_{\nu}, \alpha_{h_{1}}, \alpha_{\ell}, \alpha_{h_{2}}}} \int F_{\alpha_{h_{1}}}^{h_{1}}\left(\vec{p}_{h_{1}}\right) d^{3} \vec{p}_{h_{1}} \delta^{4}\left(p_{v}+p_{h_{1}}-p_{\ell}-p_{h_{2}}\right) \\
& \times\left(1-F_{\alpha_{h_{2}}}^{h_{2}}\left(\vec{p}_{h_{2}}\right)\right) d^{3} \vec{p}_{h_{2}}\left(1-F_{\alpha_{\ell}}^{\ell}\left(\vec{p}_{\ell}\right)\right) d^{3} \vec{p}_{\ell}
\end{aligned}
$$




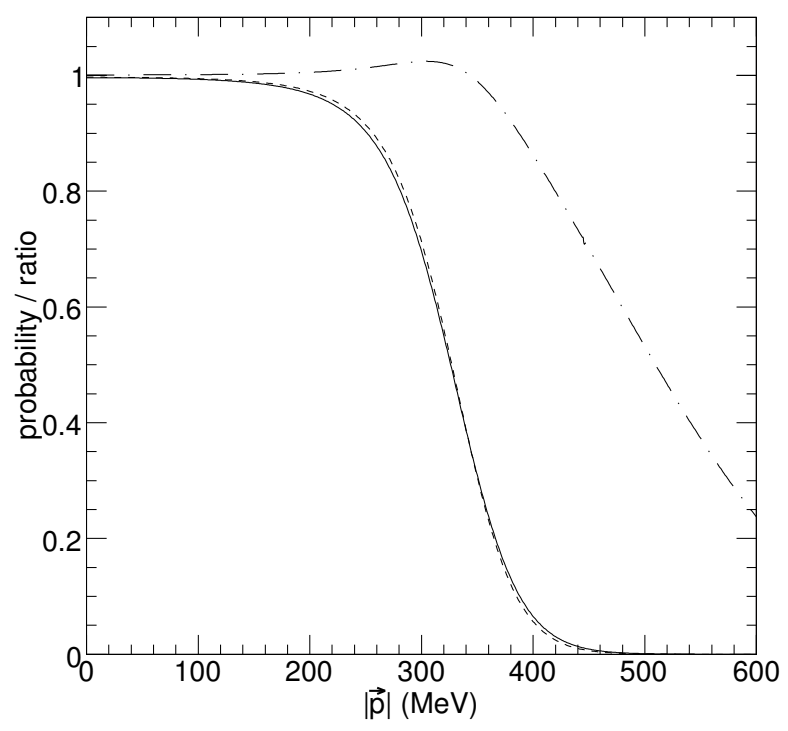

Figure 1: Comparison between a relativistic $\left(F_{R}\right.$, solid line $)$ and a non-relativistic Fermi distribution $\left(F_{N R}\right.$, dashed line), for $k T=10 \mathrm{MeV}$ and $n=0.16 \mathrm{fm}^{-3}$. The dash-dotted line represents the ratio $F_{N R} / F_{R}$.

This quantity gives then rise to the dynamic form factor $S(\omega,|\vec{q}|)$ by casting (2.2) in the following form,

$$
\frac{d^{3} \Phi}{d\left|\vec{p}_{x}\right| d^{2} \Omega_{x}}=F_{\alpha_{x}}^{\prime x}\left(\vec{p}_{x}\right)\left|\vec{p}_{x}\right|^{2} S(\omega,|\vec{q}|)
$$

\section{Results}

When considering the effects of relativity, our study shows that the larger differences are caused by the relativistic description of the Fermi distribution and not by the implementation of relativity in the dynamics of the neutrino-nucleon interaction. As Fig. 1 illustrates, it is clear that starting from the Fermi momenta, the relativistic Fermi distribution $F(p)=[\exp ((E(p)-\mu) / k T)+1]^{-1}$ obtains a slightly larger weight than its non-relativistic counter-part. This difference is especially seen in the tail of the distribution and is affected by the difference between the relativistic $E(p)=\sqrt{p^{2}+m^{2}}$ and non-relativistic $E(p)=p^{2} / 2 m$ energy expressions. It can then be translated in the following equation,

$$
\int_{p_{-}}^{\infty} F_{R}(p) p^{2} d p \geq \int_{p_{-}}^{\infty} F_{N R}(p) p^{2} d p .
$$

As can be appreciated from Fig. 2, this relation ensures that the dynamic form factor is larger when computed in a relativistic approach. It can be shown that the influence of relativity mainly enhances the dynamic form factor, while at higher momenta transfer a secondary effect occurs that shifts the maximum of the dynamic form factor to higher energy exchange [6]. Also when looking at the non-degenerate limit, relativistic corrections have to be taken into account. 


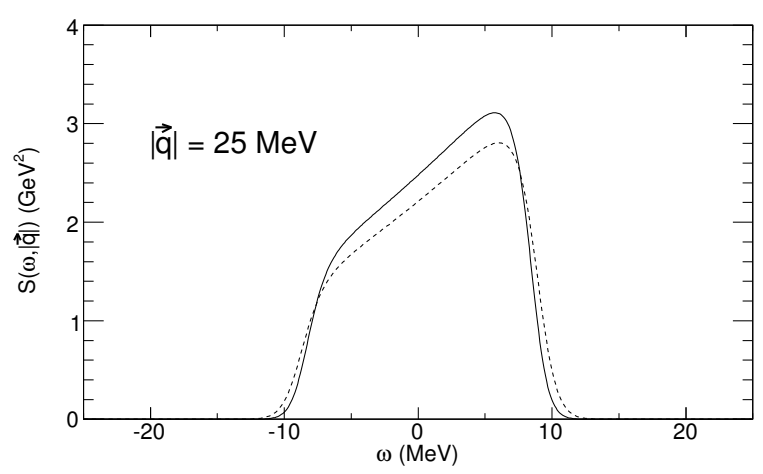

Figure 2: The dynamical form factor $S(\omega,|\vec{q}|)$ for a neutron gas with density $n=0.16 \mathrm{fm}^{-3}$ at a temperature of $k T=10 \mathrm{MeV}$, for a momentum transfer $|\vec{q}|$ of $25 \mathrm{MeV}$. The full line represents the relativistic calculation, the dashed line the non-relativistic one.

Given the minor role of relativity in the dynamics of the interaction, it is clear that the relativistic effects in the dynamic form factor will reflect themselves also in the cross section for interactions in the gas as well as in the neutrino opacities.

Although the differences in the energy distributions are relatively small (fig. 1), the energy sensitivity of the cross sections (2.1), rising fast with increasing energies, ensures that the relativistic effects have a sizable influence on the interactions under study. Relativistic cross sections are generally larger than their non-relativistic counterparts. Fig. 3 indeed shows that the opacities obtained within a relativistic calculation are considerably smaller than the ones of the non-relativistic study of Ref. [2].

A detailed description on the influence of these relativistic effects as well as on as well as the polarisation effects neutrinos have, can be found in Ref. [6]. At present, the model is improved by implementing nuclear correlations into the process.

\section{References}

[1] R. Buras, M. Rampp, H. T. Janka and K. Kifonidis, Phys. Rev. Lett. 90, 241101 (2003)

[2] S. Reddy, M. Prakash and J.M. Lattimer, Phys. Rev. D 58, 013009 (1998)

[3] A. Burrows and R.F. Sawyer, Phys. Rev. D 58, 554 (1998)

[4] C.J. Horowitz and K. Wehrberger, Nucl. Phys. A 531, 665 (1991)

[5] C.H. Llewellyn Smith, Phys. Rep. 3C, (1972)

[6] K. Vantournhout, N. Jachowicz, and J. Ryckebusch, nucl-th/0511067 


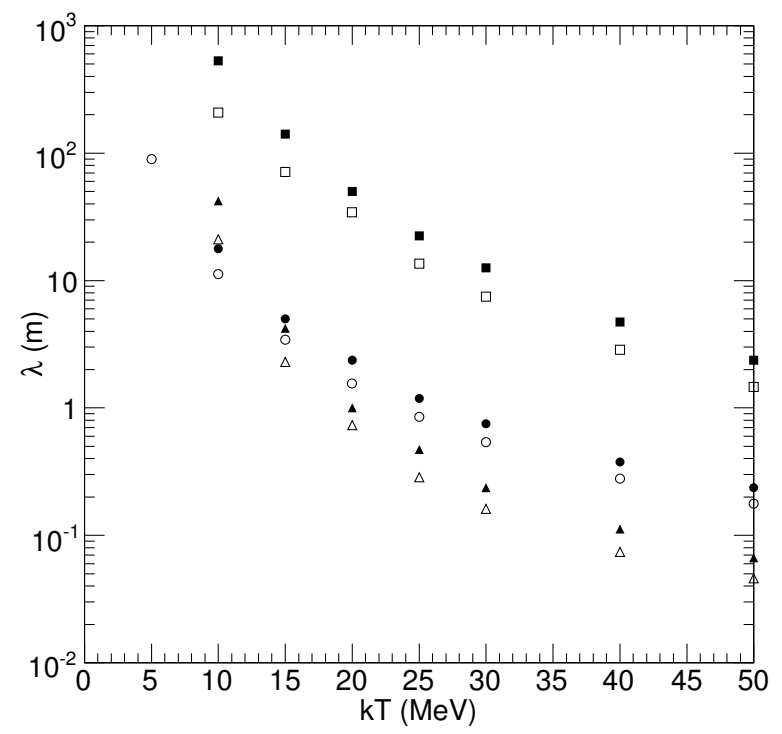

Figure 3: Neutrino mean free path as a function of the temperature for a neutron gas in beta equilibrium without neutrino trapping and $n=0.16 \mathrm{fm}^{-3}$. All results are for an initial neutrino energy of $3 k T$. The open markers present results of our relativistic calculation and are compared to the non-relativistic results of Ref. [2] (filled markers). The squares represent the mean free path for neutral-current scattering off protons $(v+p \rightarrow v+p)$, circles for neutral-current interactions with neutrons $(v+n \rightarrow v+n)$, and the triangles for the charged-current neutrino-interaction $\left(v+n \rightarrow p+e^{-}\right)$. 